\title{
Mechanical Mathematical Modelling of a Car Accident Caused by Sudden Mechanical Failure
}

\author{
Hristo Uzunov, Silvia Dechkova*, Kaloyan Dimitrov and Vasil Uzunov
}

Technical University of Sofia, Faculty and College - Sliven, Bulgaria

Received 09 August 2021; Accepted 2 October 2021

\begin{abstract}
Design of modern automobiles focuses greatly on passenger safety in vehicle cabin by creating intelligent systems to control dynamically changing parameters, as it is the case with road-and-tire friction coefficient. Problems surrounding the occurrence of sudden technical malfunction in the vehicle and the control of its behaviour after collision seem to be extremely complicated. In these circum-stances, it is basically relied on driver professional skills and their ability to maintain sustainable vehicle behaviour applying existing active and passive safety systems. Such accidents cannot be predicted in time and therefore it is good to analyze them in dynamic aspect as well as to model the expected unwanted changes in motion direction. This article offers a dynamic vehicle model with six generalized coordinates. It takes into account car suspension, damping of shock absorbers, the variable road-and-tire friction coefficient as a function of the contact point speed, the car motion in forced idling of the engine. Based on the established parameters a process of occurrence of mechanical failure has been carried out in an element of suspension system and in braking system. The model investigates undesirable car behaviour and possibilities for counteraction by controlling and handling the vehicle.
\end{abstract}

Keywords: Automobile, Active suspension, Dynamic model, Failure, Matlab.

\section{Introduction}

In dynamic investigation of road accidents, it is technically possible for the main cause of vehicle loss of lateral stability to be due to a reason beyond driver's technical skills to control the vehicle (Daily [1], Schmidt [2], Sharma [3], Wach [4]). In such cases, based on the inspection of the vehicle after collision, and the scene of collision, it is quite common to look for signs of possible sudden malfunction in vehicle suspension and braking system. This article discusses cases in which damping properties of a shock absorber suddenly change. On this basis, an assessment is made on the extent to which such a mechanical malfunction affects the change of the steering angle and the motion direction after the unexpected and sudden accident. Thus, a possible driver correction actions can be foreseen, such as turning the steering wheel or doing something else so as to come to a smooth stop (Jiang [5], Niehoff [6], Owsiański [7], Stronge [8]).

\section{Dynamic model of active car suspension}

Macro simulation of vehicle motion in case of loss of lateral stability is observed in an arbitrarily accepted absolute coordinate system $O X Y Z$ (Dechkova [9], Karapetkov [10, 11, $12,13,14])$. To study the car motion, it has been assumed that its own coordinate system $C x^{\prime} y^{\prime} z^{\prime}$ is movable and permenantly connected to the vehicle center of mass $C$ (Fig. 1). In addition, a permanently connected $C x y z$ coordinate

*E-mail address: sdechkova@tu-sofia.bg

ISSN: 1791-2377 @ 2021 School of Science, IHU. All rights reserved.

doi:10.25103/jestr.144.08 system is attached to it, parallel to the absolute and translationally movable one.

Coordinates of the vehicle center of mass $C x_{c}, y_{c}, z_{c}$ in the fixed coordinate system are selected for generalized coordinates of the car motion.

Rotational motion of the car is expressed by the Euler transformations and corresponding angles, namely $\psi, \theta$ and $\varphi$. The precession angle of $\psi$, taking into account the rotation around the axis $\mathrm{Cz}$; respectively, the angular velocity of $\dot{\psi}$ is obtained; the angle $\theta$ of nutation, taking into account the rotation with respect to the axis $C \rho$, the intersection of the planes $0 x y$ and $C x^{\prime} y$.

Therefore, the force of gravity $\vec{G}$ will lie on the axis $O z$. The spatial arrangement model of the car is a plane located on four elastic supports, which are marked by $K_{i}(i=1 \div$ 4) (Fig.2).

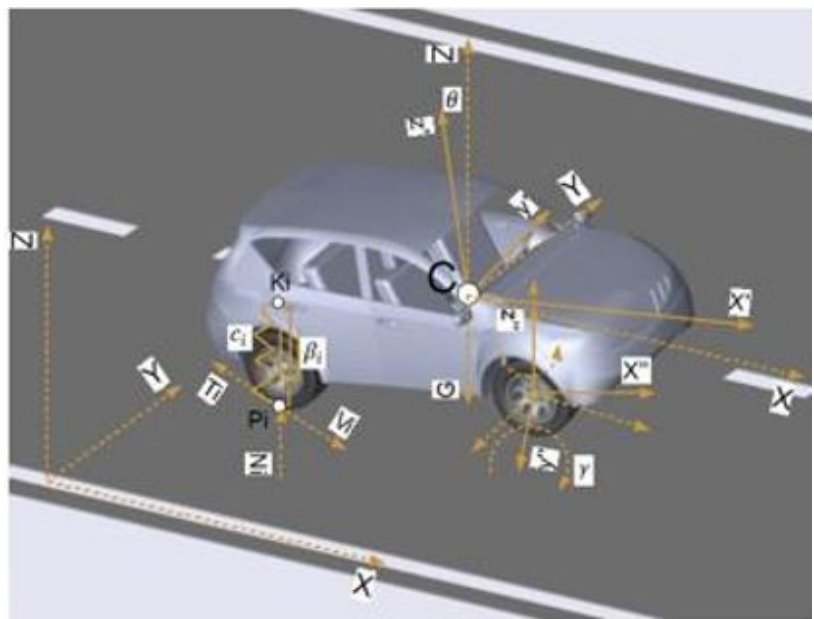

Fig. 1. Spatial dynamic model of an automobile with elastic suspension 
Hristo Uzunov, Silvia Dechkova, Kaloyan Dimitrov and Vasil Uzunov/

Journal of Engineering Science and Technology Review 14 (4) (2021) 61 - 68

$\vec{F}_{i}(i=1 \div 4)$ is elastic force generated by the elasticity of tires and springs; $\vec{N}_{i}(i=1 \div 4)$ is normal reaction at the contact point of automobile tires, corresponding to elastic force; $\vec{V}_{i}(i=1 \div 4)$ is velocity of the contact point $P_{i}$ in the plane of the road $0 x y ; \vec{T}_{i}(i=1 \div 4)$ is friction force at the contact points that lies in the plane of the road $0 x y$; $\vec{R}_{i}(i=1 \div 4)$ is resistance force generated by damping elements in suspension; $c_{i}, \frac{N}{m}(i=1 \div 4)$ elasticity of suspension, taking into account both coefficient of elasticity of tires and suspension; $b_{i}, \frac{N \cdot s}{m}(i=1 \div 4)$ coefficient of linear resistance.

The car motion according to the studies of kinetic energy and generalized forces is defined by six differential equations with six generalized coordinates. These equations are valid if the friction force is in accordance with Coulomb's law and the wheels slide on the ground without rolling. According to (5), the wheels keep a continuous contact with the road.
Generalized forces and moments in the right-hand sides of the differential equations (1) are determined by assuming that the absolute coordinate system has a vertical axis of $\mathrm{O} z$.

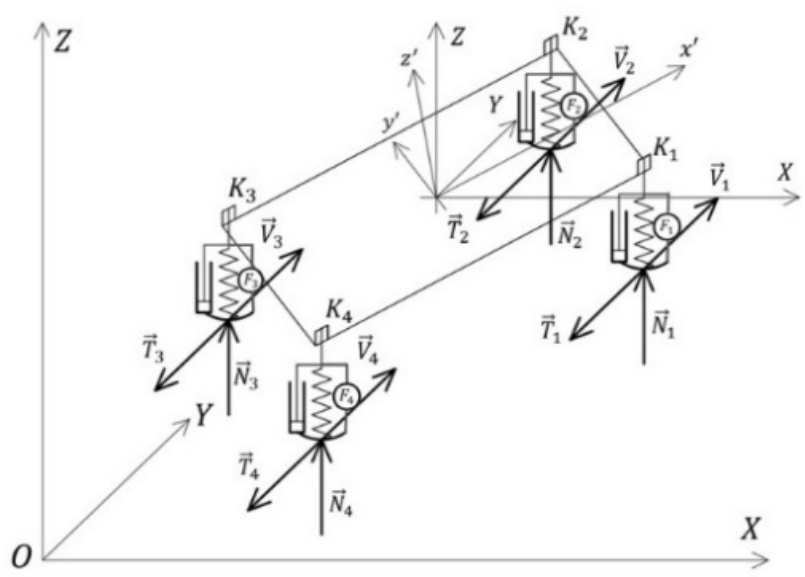

Fig. 2. Model of the forces acting on a car in its spatial motion, taking into account the elasticity of tires (suspension).

$$
m \cdot \ddot{x}=\left[\sum_{i=1}^{4} F_{x i}\right] ; m \cdot \ddot{y}=\left[\sum_{i=1}^{4} F_{y i}\right] ; m \cdot \ddot{z}=\left[-G+\sum_{i=1}^{4} N_{i}-\sum_{i=1}^{4} R_{i}\right]
$$

$a_{11} \cdot \ddot{\varphi}+a_{12} \cdot \ddot{\psi}+a_{13} \cdot \ddot{\theta}=\left\{\begin{array}{c}\sum_{i=1}^{4} N_{i} \cdot \delta_{\varphi i}+\sum_{i=1}^{4}\left(F_{x i} \cdot f_{\varphi_{x}}+F_{y i} \cdot f_{\varphi_{y}}\right)-\sum_{i=1}^{4} R_{i} \cdot \delta_{\varphi i} \\ -b_{11} \cdot \dot{\varphi}^{2}-b_{12} \cdot \dot{\psi}^{2}-b_{13} \cdot \dot{\theta}^{2}-c_{11} \cdot \dot{\varphi} \cdot \dot{\psi}-c_{12} \cdot \dot{\varphi} \cdot \dot{\theta}-c_{13} \cdot \dot{\psi} \cdot \dot{\theta}\end{array}\right\}$

$a_{21} \cdot \ddot{\varphi}+a_{22} \cdot \ddot{\psi}+a_{23} \cdot \ddot{\theta}=\left\{\begin{array}{c}\sum_{i=1}^{4}\left(F_{x i} \cdot f_{\psi_{x}}+F_{y i} \cdot f_{\psi_{y}}\right)-b_{21} \cdot \dot{\varphi}^{2}-b_{22} \cdot \dot{\psi}^{2}-b_{23} \cdot \dot{\theta}^{2}- \\ -c_{21} \cdot \dot{\varphi} \cdot \dot{\psi}-c_{22} \cdot \dot{\varphi} \cdot \dot{\theta}-c_{23} \cdot \dot{\psi} \cdot \dot{\theta}\end{array}\right\}$

$a_{31} \cdot \ddot{\varphi}+a_{32} \cdot \ddot{\psi}+a_{33} \cdot \ddot{\theta}=\left\{\begin{array}{c}\sum_{i=1}^{4} N_{i} \cdot \delta_{\theta i}+\sum_{i=i}^{4}\left(F_{x i} \cdot f_{\theta_{x}}+F_{y i} \cdot f_{\theta_{y}}\right)-\sum_{i=1}^{4} R_{i} \cdot \delta_{\theta i}- \\ -b_{31} \cdot \dot{\varphi}^{2}-b_{32} \cdot \dot{\psi}^{2}-b_{33} \cdot \dot{\theta}^{2}-c_{31} \cdot \dot{\varphi} \cdot \dot{\psi}-c_{32} \cdot \dot{\varphi} \cdot \dot{\theta}-c_{33} \cdot \dot{\psi} \cdot \dot{\theta}\end{array}\right\}$

$a_{11}=J_{z^{\prime} z^{\prime}} ; a_{12}=-J_{z^{\prime} z^{\prime}} \cdot \cos \theta-J_{z^{\prime} x^{\prime}} \cdot \sin \varphi \cdot \sin \theta-J_{y^{\prime} z^{\prime}} \cdot \cos \varphi \cdot \sin \theta ;$

$a_{13}=-J_{z^{\prime} x^{\prime}} \cdot \cos \varphi+J_{y^{\prime} z^{\prime}} \cdot \sin \varphi ;$

$b_{11}=0 ; b_{12}=\left(\begin{array}{c}-\frac{1}{2} \cdot \sin 2 \varphi \cdot \sin ^{2} \theta \cdot\left(J_{x^{\prime} x^{\prime}}+J_{y^{\prime} y^{\prime}}\right)+ \\ +J_{x^{\prime} y^{\prime}} \cdot \cos 2 \varphi \cdot \sin ^{2} \theta+ \\ +\frac{1}{2} \cdot \sin 2 \theta \cdot\left(J_{z^{\prime} x^{\prime}} \cdot \cos \varphi-J_{y^{\prime} z^{\prime}} \cdot \sin \varphi\right)\end{array}\right)$;

$b_{13}=\left(\frac{1}{2} \cdot\left(J_{x^{\prime} x^{\prime}}-J_{y^{\prime} y^{\prime}}\right) \cdot \sin 2 \varphi-J_{x^{\prime} y^{\prime}} \cdot \cos 2 \varphi\right)$;

$c_{11}=0 ; \quad c_{12}=0 ; c_{13}=\left(\begin{array}{c}\cos 2 \varphi \cdot \sin \theta \cdot\left(J_{x^{\prime} x^{\prime}}+J_{y^{\prime} y^{\prime}}\right)- \\ -J_{z^{\prime} z^{\prime}} \cdot \sin \theta- \\ -2 \cdot\left(\begin{array}{c}J_{x^{\prime} y^{\prime}} \cdot \sin 2 \varphi \cdot \sin \theta+ \\ +J_{z^{\prime} x^{\prime}} \cdot \sin \varphi \cdot \cos \theta+ \\ +J_{y^{\prime} z^{\prime}} \cdot \cos \varphi \cdot \cos \theta\end{array}\right)\end{array}\right)$

$a_{21}=\left(J_{z^{\prime} z^{\prime}} \cdot \cos \theta-J_{z^{\prime} x^{\prime}} \cdot \sin \varphi \cdot \sin \theta-J_{y^{\prime} z^{\prime}} \cdot \cos \varphi \cdot \sin \theta\right) ;$

$a_{22}=\left(\begin{array}{c}J_{x^{\prime} x^{\prime}} \cdot \sin ^{2} \varphi \cdot \sin ^{2} \theta+J_{y^{\prime} y^{\prime}} \cdot \cos ^{2} \varphi \cdot \sin ^{2} \theta+ \\ +J_{z^{\prime} z^{\prime}} \cdot \cos ^{2} \theta-J_{x^{\prime} y^{\prime}} \cdot \sin 2 \varphi \cdot \sin ^{2} \theta \\ -J_{x^{\prime} z^{\prime}} \cdot \sin \varphi \cdot \sin 2 \theta-J_{y^{\prime} z^{\prime}} \cdot \cos \varphi \cdot \sin 2 \theta\end{array}\right) ;$ 


$$
\begin{aligned}
& a_{23}=\left(\begin{array}{c}
0,5 \cdot J_{x^{\prime} x^{\prime}} \cdot \sin 2 \varphi \cdot \sin \theta-\frac{1}{2} \cdot J_{y^{\prime} y^{\prime}} \cdot \sin 2 \varphi \cdot \sin \theta- \\
-J_{x^{\prime} y^{\prime}} \cdot \cos 2 \varphi \cdot \sin \theta-J_{z^{\prime} x^{\prime}} \cdot \cos \varphi \cdot \cos \theta+ \\
+J_{y^{\prime} z^{\prime}} \cdot \sin \varphi \cdot \cos \theta
\end{array}\right) \\
& b_{21}=\left(-J_{z^{\prime} x^{\prime}} \cdot \cos \varphi+J_{y^{\prime} z^{\prime}} \cdot \sin \varphi\right) \cdot \sin \theta \\
& b_{22}=0 ; b_{23}=\left(\begin{array}{c}
0,5 \cdot J_{x^{\prime} x^{\prime}} \cdot \sin 2 \varphi-\frac{1}{2} \cdot J_{y^{\prime} y^{\prime}} \cdot \sin 2 \varphi- \\
-J_{x^{\prime} y^{\prime}} \cdot \cos 2 \varphi \\
+\left(J_{z^{\prime} x^{\prime}} \cdot \cos \varphi-J_{y^{\prime} z^{\prime}} \cdot \sin \varphi\right) \cdot \sin \theta
\end{array}\right) \cdot \cos \theta+ \\
& c_{21}=\left(\begin{array}{c}
\left(J_{x^{\prime} x^{\prime}} \cdot \sin 2 \varphi-J_{y^{\prime} y^{\prime}} \cdot \sin 2 \varphi-2 \cdot J_{x^{\prime} y^{\prime}} \cdot \cos 2 \varphi\right) \cdot \sin ^{2} \theta- \\
-\left(J_{z^{\prime} x^{\prime}} \cdot \cos \varphi-J_{y^{\prime} z^{\prime}} \cdot \sin \varphi\right) \cdot \sin 2 \theta
\end{array}\right) \\
& c_{22}=\left(\begin{array}{c}
\left(J_{x^{\prime} x^{\prime}} \cdot \cos 2 \varphi-J_{y^{\prime} y^{\prime}} \cdot \cos 2 \varphi+2 \cdot J_{x^{\prime} y^{\prime}} \cdot \sin 2 \varphi\right) \cdot \sin \theta- \\
-J_{z^{\prime} z^{\prime}} \cdot \sin \theta
\end{array}\right) ; \\
& c_{23}=\left(\begin{array}{c}
\left(J_{x^{\prime} x^{\prime}} \cdot \sin ^{2} \varphi+J_{y^{\prime} y^{\prime}} \cdot \cos ^{2} \varphi-J_{x^{\prime} y^{\prime}} \cdot \sin 2 \varphi-J_{z^{\prime} z^{\prime}}\right) \cdot \sin 2 \theta- \\
-2 \cdot\left(J_{z^{\prime} x^{\prime}} \cdot \sin \varphi+J_{y^{\prime} z^{\prime}} \cdot \cos \varphi\right) \cdot \cos 2 \theta
\end{array}\right) \\
& a_{31}=J_{z^{\prime} x^{\prime}} \cdot \cos \varphi+J_{y^{\prime} z^{\prime}} \cdot \sin \varphi \\
& a_{32}=\left[\begin{array}{c}
0,5 \cdot\left(J_{x^{\prime} x^{\prime}}-J_{y^{\prime} y^{\prime}}\right) \cdot \sin 2 \varphi \cdot \sin \theta-J_{x^{\prime} y^{\prime}} \cdot \cos 2 \varphi \cdot \sin \theta- \\
-J_{z^{\prime} x^{\prime}} \cdot \cos \varphi \cdot \cos \theta+J_{y^{\prime} z^{\prime}} \cdot \sin \varphi \cdot \cos \theta
\end{array}\right] \\
& a_{33}=J_{x^{\prime} x^{\prime}} \cdot \cos ^{2} \varphi+J_{y^{\prime} y^{\prime}} \cdot \sin ^{2} \varphi+\frac{1}{2} \cdot J_{x^{\prime} y^{\prime}} \cdot \sin 2 \varphi ; \quad b_{31}=J_{z^{\prime} x^{\prime}} \cdot \sin \varphi+J_{y^{\prime} z^{\prime}} \cdot \cos \varphi \\
& b_{32}=\left[\begin{array}{c}
-\left[0,5 \cdot\left(\begin{array}{c}
\left.J_{x^{\prime} x^{\prime}} \cdot \sin ^{2} \varphi+J_{y^{\prime} y^{\prime}} \cdot \cos ^{2} \varphi+\right) \\
+J_{z^{\prime} z^{\prime}}-J_{x^{\prime} y^{\prime}} \cdot \sin 2 \varphi
\end{array}\right)\right] \cdot \sin 2 \theta+ \\
+\left(J_{z^{\prime} x^{\prime}} \cdot \sin \varphi+J_{y^{\prime} z^{\prime}} \cdot \cos \varphi\right) \cdot \cos 2 \theta
\end{array}\right] ; \quad b_{33}=0 ; \\
& c_{31}=\left[\begin{array}{c}
{\left[\left(J_{x^{\prime} x^{\prime}}+J_{y^{\prime} y^{\prime}}\right) \cdot \cos 2 \varphi+2 \cdot J_{x^{\prime} y^{\prime}} \cdot \sin 2 \varphi+J_{z^{\prime} z^{\prime}}\right] \cdot \sin \theta+} \\
+2 \cdot\left(J_{z^{\prime} x^{\prime}} \cdot \sin \varphi+J_{y^{\prime} z^{\prime}} \cdot \cos \varphi\right) \cdot \cos \theta
\end{array}\right] \text {; } \\
& c_{32}=\left[\left(-J_{x^{\prime} x^{\prime}}+J_{y^{\prime} y^{\prime}}\right) \cdot \sin 2 \varphi+2 \cdot J_{x^{\prime} y^{\prime}} \cdot \cos 2 \varphi\right] ; c_{33}=0
\end{aligned}
$$

We substitute the equations before $\delta_{\varphi i}$ and $\delta_{\theta i}$ using the notation

$$
\begin{aligned}
\delta_{\varphi i} & =\left[(\cos \varphi \cdot \sin \theta) \cdot x_{k i}^{\prime}+(-\sin \varphi \cdot \sin \theta) \cdot y_{k i}^{\prime}\right] \\
\delta_{\theta i} & =\left[\begin{array}{c}
(\sin \varphi \cdot \cos \theta) \cdot x_{k i}^{\prime}+ \\
+(\cos \varphi \cdot \cos \theta) \cdot y_{k i}^{\prime}+(-\sin \theta) \cdot z_{k i}^{\prime}
\end{array}\right]
\end{aligned}
$$

To facilitate notation, substitution has been done, which looks like as follows:

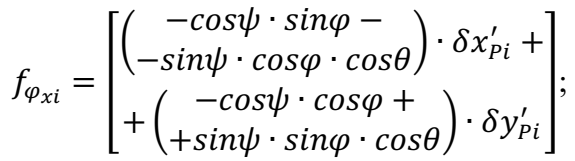

$$
\begin{aligned}
& f_{\psi_{x i}} \\
& =\left[\begin{array}{c}
(-\sin \psi \cdot \cos \varphi-\cos \psi \cdot \sin \varphi \cdot \cos \theta) \cdot \delta x_{k i}^{\prime}+ \\
+(\sin \psi \cdot \sin \varphi-\cos \psi \cdot \cos \varphi \cdot \cos \theta) \cdot \delta y_{k i}^{\prime}+ \\
+(-\cos \psi \cdot \sin \theta) \cdot \delta z_{k i}^{\prime}
\end{array}\right] ;
\end{aligned}
$$

$$
\begin{aligned}
& f_{\theta_{x i}}=\left[\begin{array}{c}
(\sin \theta \cdot \sin \psi \cdot \sin \varphi) \cdot \delta x_{k i}^{\prime}+ \\
+(\sin \theta \cdot \sin \psi \cdot \cos \varphi) \cdot \delta y_{k i}^{\prime}+ \\
+(-\cos \theta \cdot \sin \psi) \cdot \delta z_{k i}^{\prime}
\end{array}\right] \\
& f_{\varphi_{y i}}=\left[\begin{array}{c}
\left(\begin{array}{c}
-\sin \psi \cdot \sin \varphi+ \\
+\cos \psi \cdot \sin \varphi \cdot \cos \theta
\end{array}\right) \cdot \delta x_{k i}^{\prime}+ \\
+\left(\begin{array}{c}
-\sin \psi \cdot \cos \varphi- \\
-\cos \psi \cdot \sin \varphi \cdot \cos \theta
\end{array}\right) \cdot \delta y_{k i}^{\prime}
\end{array}\right] ; \\
& f_{\psi_{y i}}=\left[\begin{array}{c}
\left(\begin{array}{c}
\cos \psi \cdot \cos \varphi- \\
-\sin \psi \cdot \sin \varphi \cdot \cos \theta
\end{array}\right) \cdot \delta x_{k i}^{\prime}+ \\
+\left(\begin{array}{c}
-\cos \psi \cdot \sin \varphi- \\
-\sin \psi \cdot \cos \varphi \cdot \cos \theta
\end{array}\right) \cdot \delta y_{k i}^{\prime}+ \\
+(\sin \psi \cdot \sin \theta) \cdot \delta z_{k i}^{\prime}
\end{array}\right] \\
& f_{\theta_{y i}}=\left[\begin{array}{c}
(-\cos \psi \cdot \sin \varphi \cdot \sin \theta) \cdot \delta x_{k i}^{\prime}+ \\
+(-\cos \psi \cdot \cos \varphi \cdot \sin \theta) \cdot \delta y_{k i}^{\prime}+ \\
+(-\cos \psi \cdot \sin \theta) \cdot \delta z_{k i}^{\prime}
\end{array}\right] .
\end{aligned}
$$


Hristo Uzunov, Silvia Dechkova, Kaloyan Dimitrov and Vasil Uzunov/

Journal of Engineering Science and Technology Review 14 (4) (2021) 61 - 68

The relative motion of the wheels, the differential(s) and the engine are characterized by a system of four differential equatio

$\left[I_{\gamma}\right] \cdot[\ddot{\gamma}]=\left[M_{\gamma i}\right] ;$

$M_{\gamma i}=\left\{F_{i \tau} \cdot r_{i}+\operatorname{sign}\left(\dot{\gamma}_{i}\right) \cdot\left[M_{d i}--f_{i} \cdot N_{i}-M_{s i}\right]\right\}$

$\vec{F}_{i \tau}$ is tangential component of the tire-road friction force, the positive direction of which is taken backwards, in the more frequent cases of braking or loss of stiffness.

Where $\mu$ is friction coefficient depending on slipping speed on the contact spot; $\vec{r}_{i}$ - radius of the wheel; $f_{i}-$ coefficient of rolling friction; $\vec{N}_{i}$ - normal reaction of the road on wheels; $\left[I_{\gamma}\right]$ - a square matrix of coefficients in front the actual angular acceleration of the drive wheels, depending on the moment of inertia of the wheels and the engine; $\dot{\gamma}_{i} / i=$ $1 \div 4$ / - wheel angular velocity; $[\ddot{\gamma}]$ - a matrix-column of the actual angular acceleration of the wheels, two or four of which are propulsive; $M_{d i}, M_{s i}$ - corresponding engine and brake torque applied to each wheel.

Figure 3 shows the dynamic model of an active suspension system. Figure 4 shows the dynamic diagram of a driving or sliding wheel.
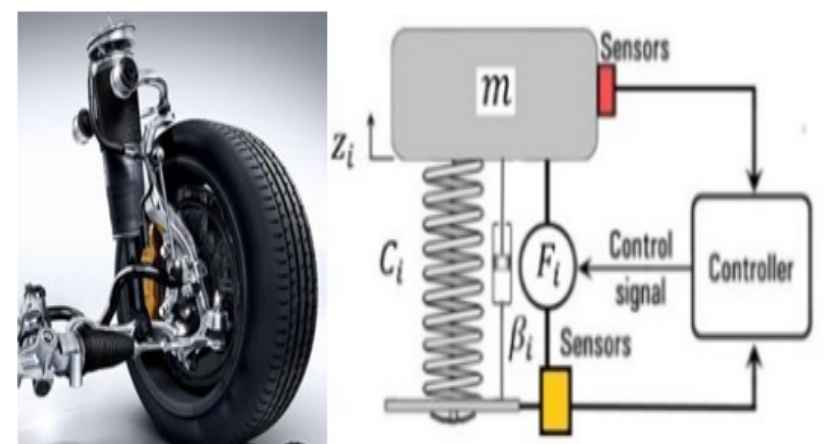

Fig. 3. Dynamic model of an active suspension system.

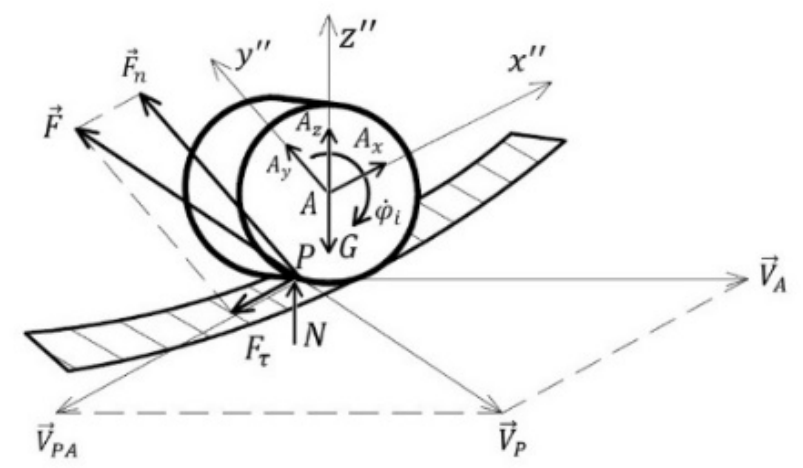

Fig. 4. Drive wheel diagram.

\section{Numerical experiment of a spatial motion vehicle model}

Mechanical and mathematical modelling of vehicle motion in the event of a sudden technical problem with suspension system of the car.

Technical data of an automobile are: mass $m=$ $1180 \mathrm{~kg}$; length $\mathrm{a}=4,31 \mathrm{~m}$; width $b=1,74 \mathrm{~m}$; longitudinal base $b=2,65 \mathrm{~m}$.

Initial linear and angular velocity of the car are as follows:
$V x=100 \mathrm{~km} / \mathrm{h}$;

$V y=0 \mathrm{~km} / \mathrm{h} ; V z=0 \mathrm{~km} / \mathrm{h}$;

$\dot{\psi}=0 s^{-1} ; \dot{\theta}=0 s^{-1} ; \dot{\varphi}=0 s^{-1}$

Elastic constants of springs without active and with active suspension are as follows:

$c_{i}=\left[\begin{array}{llll}20000 & 20000 & 18000 & 18000\end{array}\right],[\mathrm{N} / \mathrm{cm}]$

$c_{i 1}=\left[\begin{array}{llll}100000 & 100000 & 100000 & 100000\end{array}\right],[\mathrm{N} / \mathrm{cm}]$

Damping factor without active and with active suspension is as follows:

$\beta_{i}=\left[\begin{array}{llll}5246 & 5246 & 4208 & 4208\end{array}\right],[N . s / \mathrm{cm}]$

$\beta_{i 1}=\left[\begin{array}{llll}11731 & 11731 & 9919 & 9919\end{array}\right],[$ N.s $/ \mathrm{cm}]$

Numerical investigation is in case of a sudden technical problem in the front left suspension, where the shock absorber locks. The solutions of the system of differential equations of motion (1) are shown graphically (fig. 5-11).

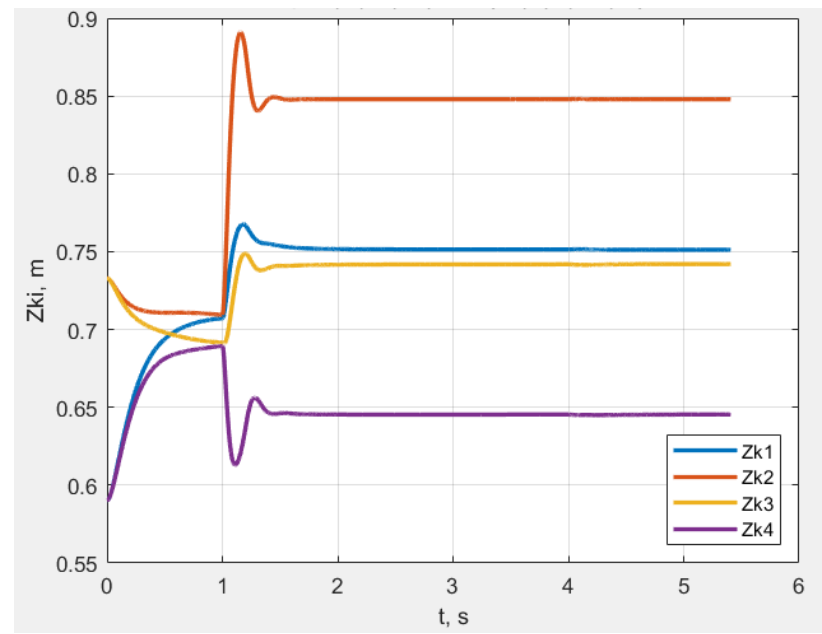

Fig. 5. Coordinates of suspension points.

\subsection{Numerical example of a failure in the brake system}

A common phenomenon in the effective application of vehicle's braking system is the difference in length between left and right brake tracks at the initial moment of effective braking.

A similar process is possible with a working brake system, but with a difference in the normal reaction in the wheels. The nature of the tracks is due to the complex process of friction between the tires and the road surface with a dynamic redistribution of pressure forces in the wheels. A brake trace is observed in accordance with Coulomb's law, in the presence of sufficiently large braking torque and coefficient of adhesion, friction force being at sliding and is less than at rest.

Coulomb's law of friction has the form

$T \leq \mu_{o} . N$

where friction force $\mathrm{T}$ does not exceed the so-called friction force at rest. The latter is proportional to the normal reaction in the wheel, respectively the pressure $\mathrm{N}$, with a coefficient of 
Hristo Uzunov, Silvia Dechkova, Kaloyan Dimitrov and Vasil Uzunov/

Journal of Engineering Science and Technology Review 14 (4) (2021) 61 - 68

proportionality $\mu_{0}$ - coefficient of friction at rest. The higher the pressure, the less likely the tire is to slip.

The above analysis excludes technical malfunction. Proof of this is the given numerical example, which examines the automobile motion with uneven braking force in the front wheels. With a sudden difference of up to $40 \%$ lower value of the braking torque in the left wheels compared to the right ones there is a rotation around a vertical axis, clockwise (fig. 12-18).

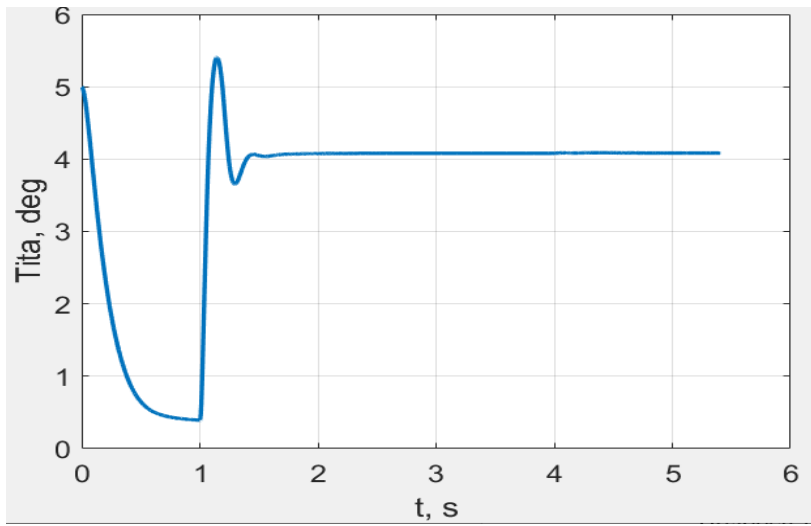

Fig. 6. Changes in the angle around the $C \rho$ axis.

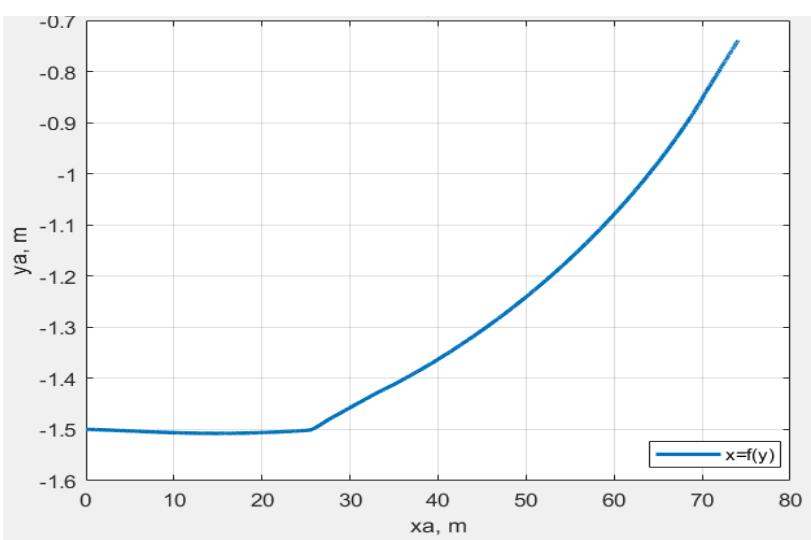

Fig. 7. Trajectory of center of mass.

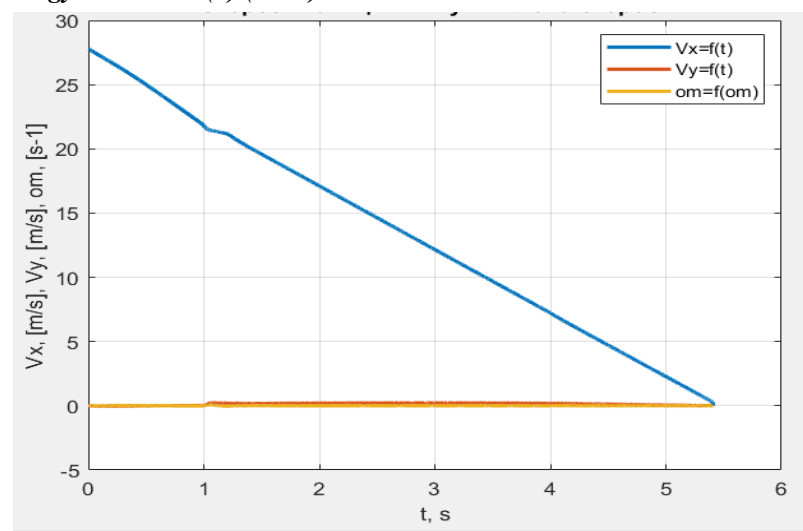

Fig. 8. Center of mass velocity and angular velocity.

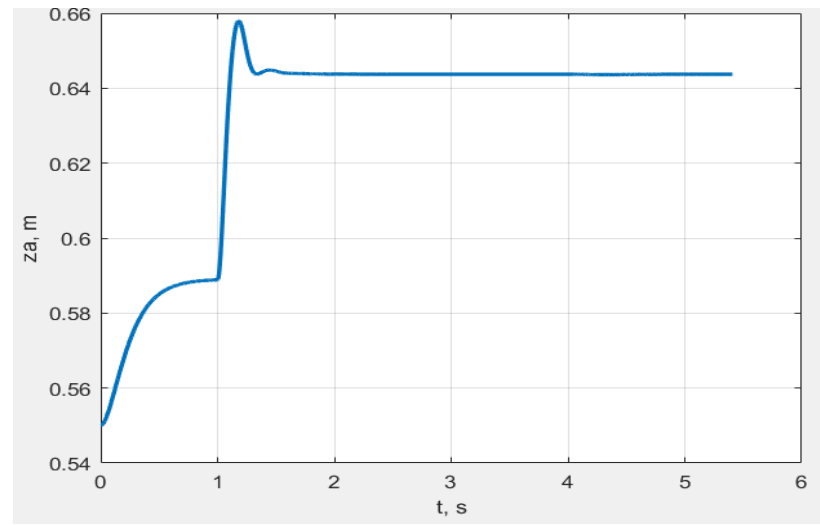

Fig. 9. Z-axis of the center of mass.

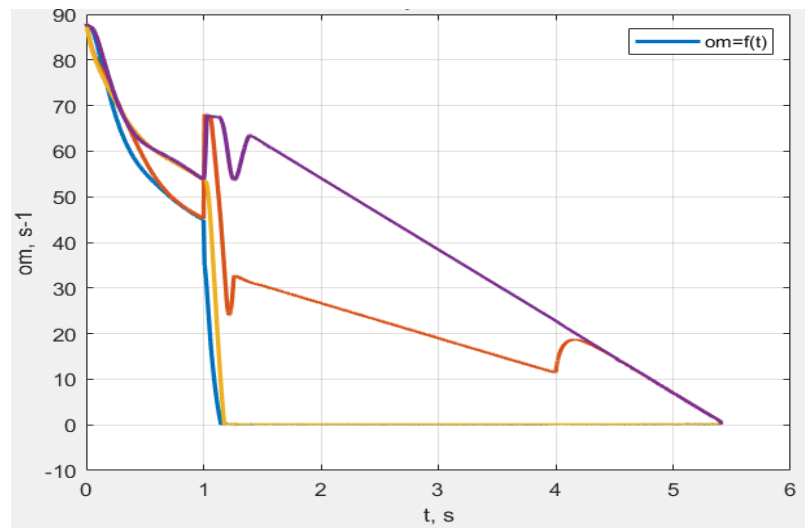

Fig. 10. Angular velocity of the wheels $\dot{\gamma}_{i}(i=1 \div 4)$.

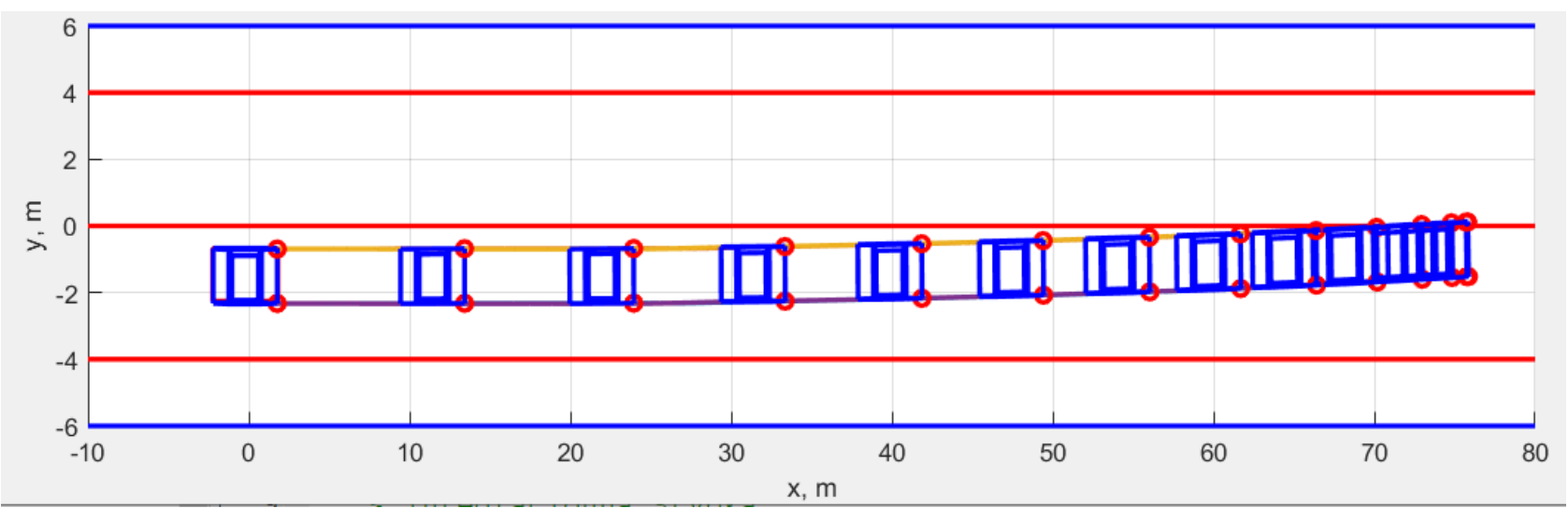

Fig. 11. Discrete positions of vehicle's motion. 
Hristo Uzunov, Silvia Dechkova, Kaloyan Dimitrov and Vasil Uzunov/

Journal of Engineering Science and Technology Review 14 (4) (2021) 61 - 68

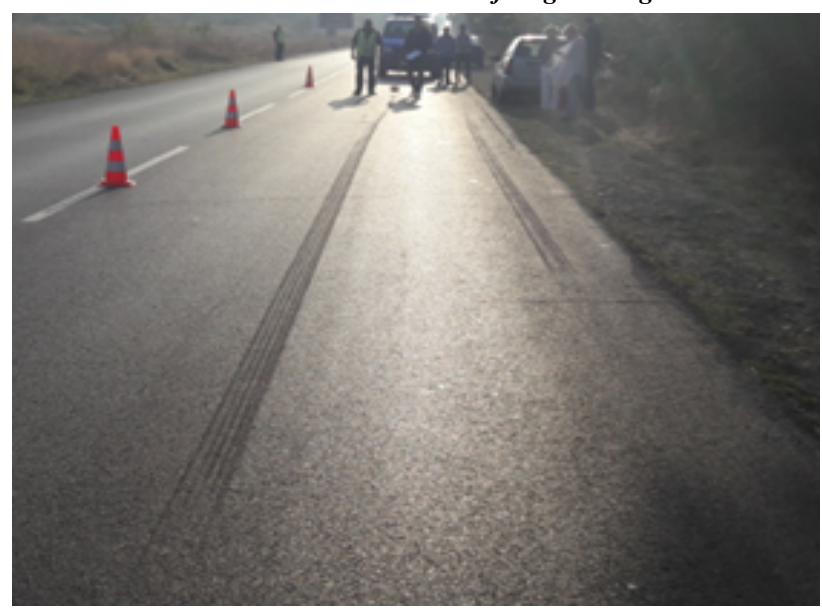

Fig. 12. Brake tracks.

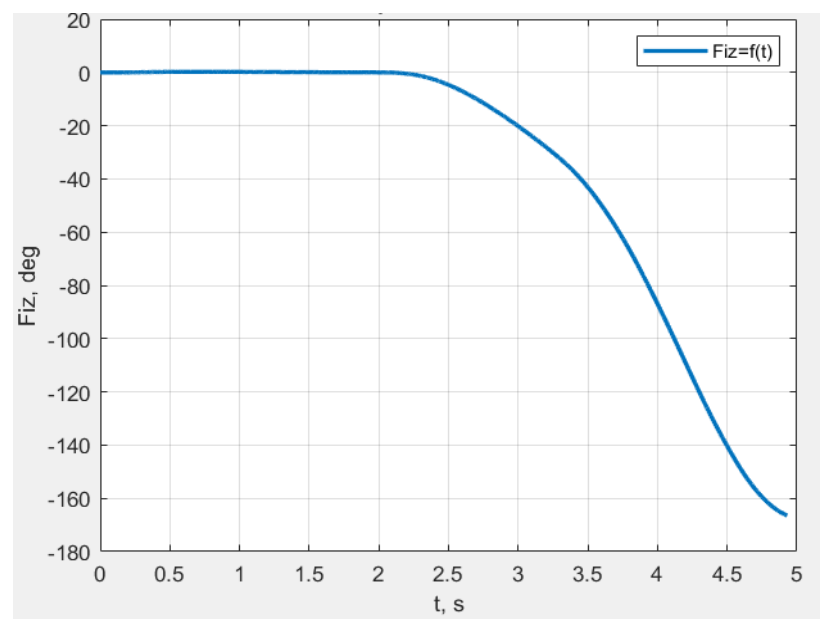

Fig. 13. Changes in the angle around the $\mathrm{Oz}$ axis.

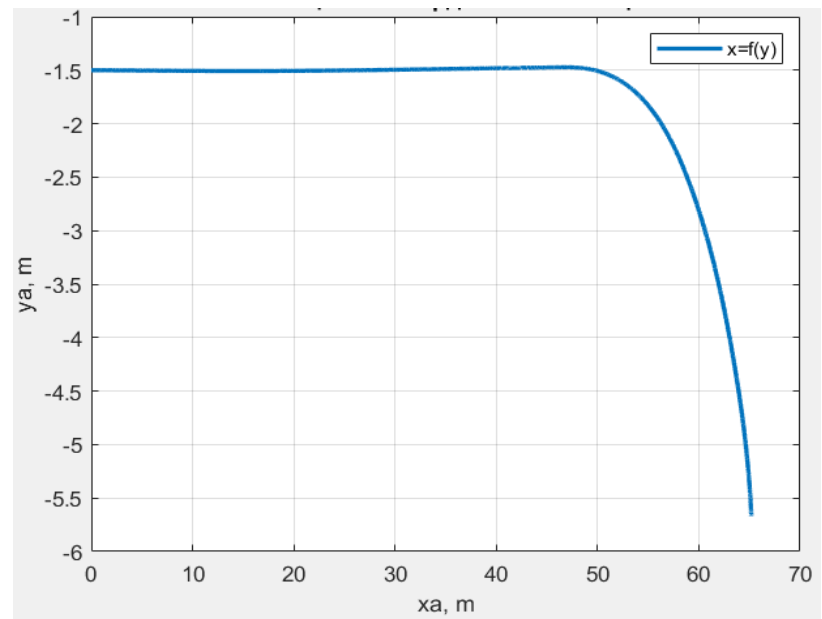

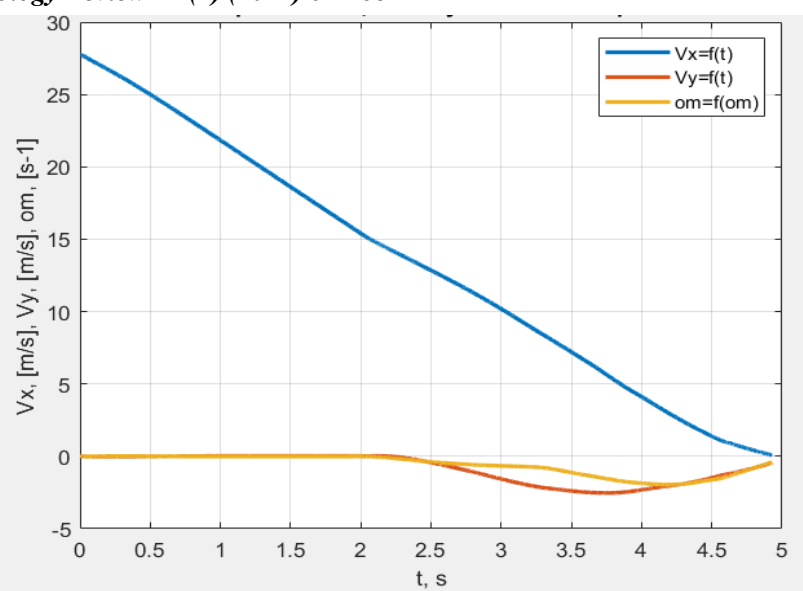

Fig. 15. Center of mass velocity and angular velocity.

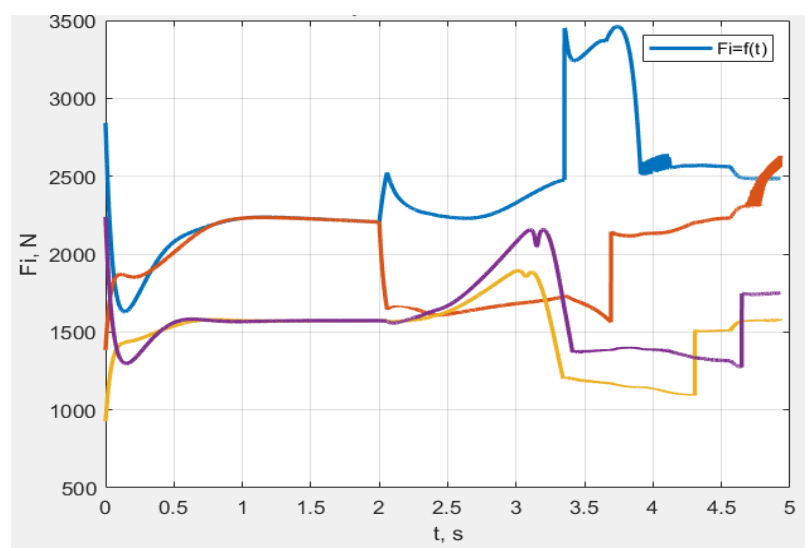

Fig.16. Road-and tire friction force $N_{1}(i=1 \div 4)$ is normal reaction.

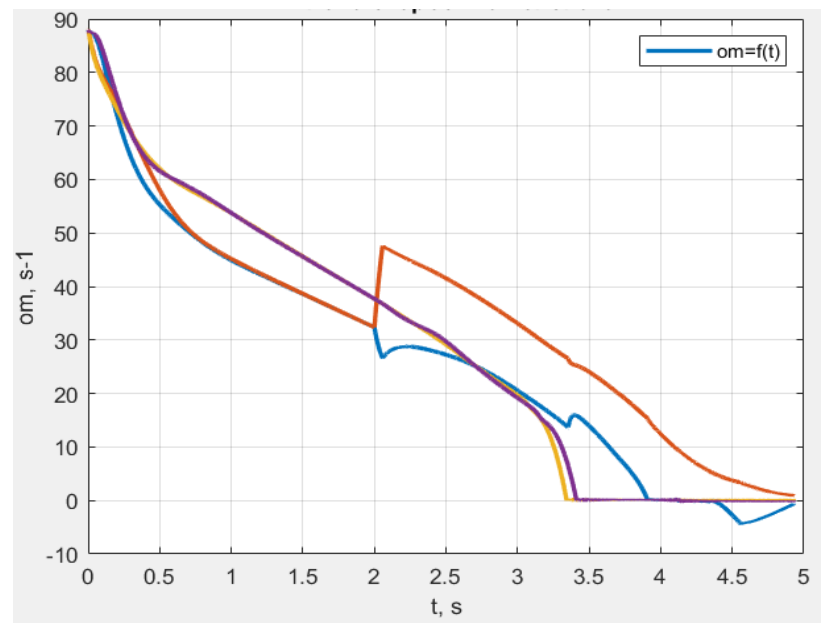

Fig. 17. Angular velocity of wheels $\dot{\gamma}_{i}(i=1 \div 4)$.

Fig. 14. Trajectory of center of mass.

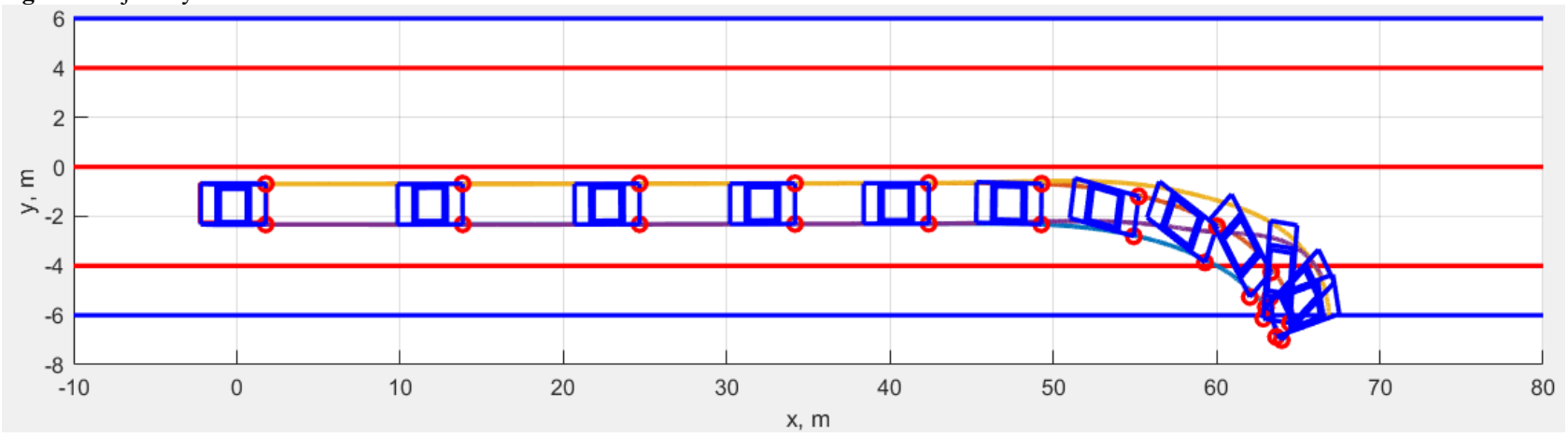

Fig. 18. Discrete positions of vehicle's motion. 
Hristo Uzunov, Silvia Dechkova, Kaloyan Dimitrov and Vasil Uzunov/

Journal of Engineering Science and Technology Review 14 (4) (2021) 61 - 68

\subsection{Sudden mechanical type failure}

When driving a car, there are cases of sudden mechanical type failures. Particularly dangerous are the ones when there is a high speed tire blowout so that the radius of the wheel changes suddenly. They are most often observed in conditions of high temperature on asphalt roads, significantly reduced criteria for tire tread depth and worn grooves, overload of the wheel, close to maximum. The study is based on the differential equations of motion for a driving wheel (5) depending on whether the drive is front, rear or $4 \times 4$.

The speed of motion of the vehicle center of mass is set at 120 $\mathrm{km} / \mathrm{h}$, and after $2 \mathrm{~s}$ there is a sudden blowout in the right front tyre (fig. 19-25).

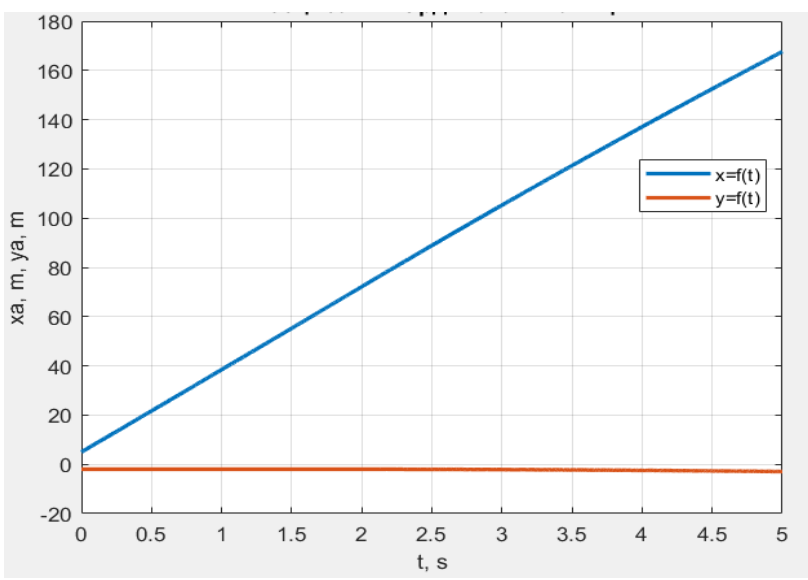

Fig. 19. Coordinates of the mass center.

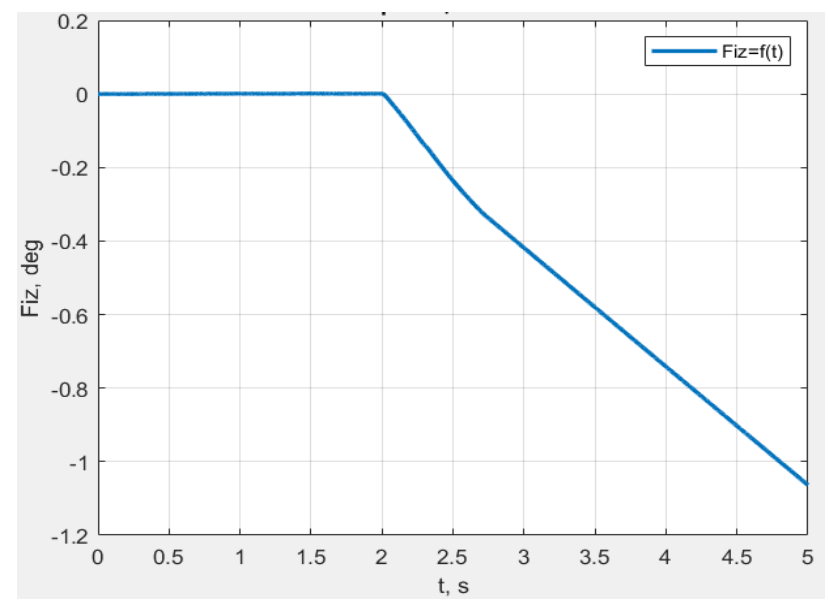

Fig. 20. Changes in the angle around the $\mathrm{Oz}$ axis.

The sudden change in the direction of the vehicle motion to the right leads to a correction of vehicle heading and steering angle of the wheels. At high center of mass velocity, this would lead to a violation of stability and loss of control.

\section{Conclusion}

1. Occurrence of damper failure leads to a change in rectilinear motion, resulting from change of the dynamos by inertial forces. From technical safety point of view, driver corrective actions should come to a smooth stop and the technical fault rectified.

2. The difference in the length of the sliding trace at the initial moment of effective braking, when turning the steering wheel, is not due to technical malfunction, but to the complex process of friction between the road and the tires with dynamic redistribution of pressure forces in the wheels.

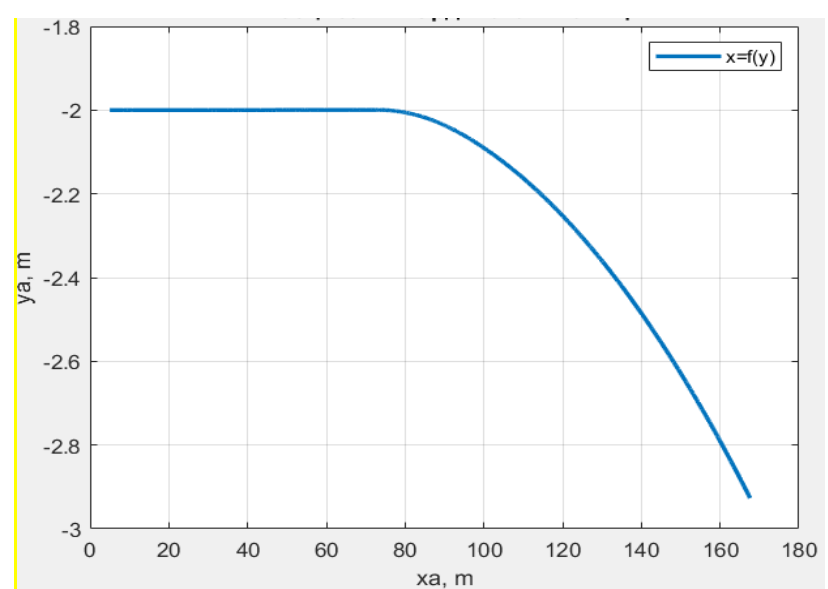

Fig. 21. Trajectory of center of mass.

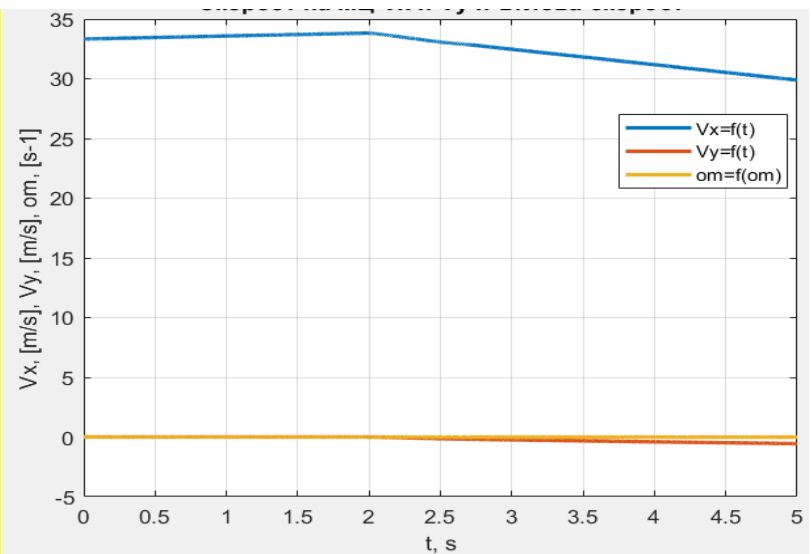

Fig. 22. Center of mass velocity and angular velocity.

3. In the event of a sudden difference in braking torque in the front wheels up to $40 \%$, there is a loss of lateral stability and rotation of the vehicle around a vertical axis. Driving the vehicle in the event of a sudden technical failure in the front wheel during an emergency stop leads to a loss of lateral stability.

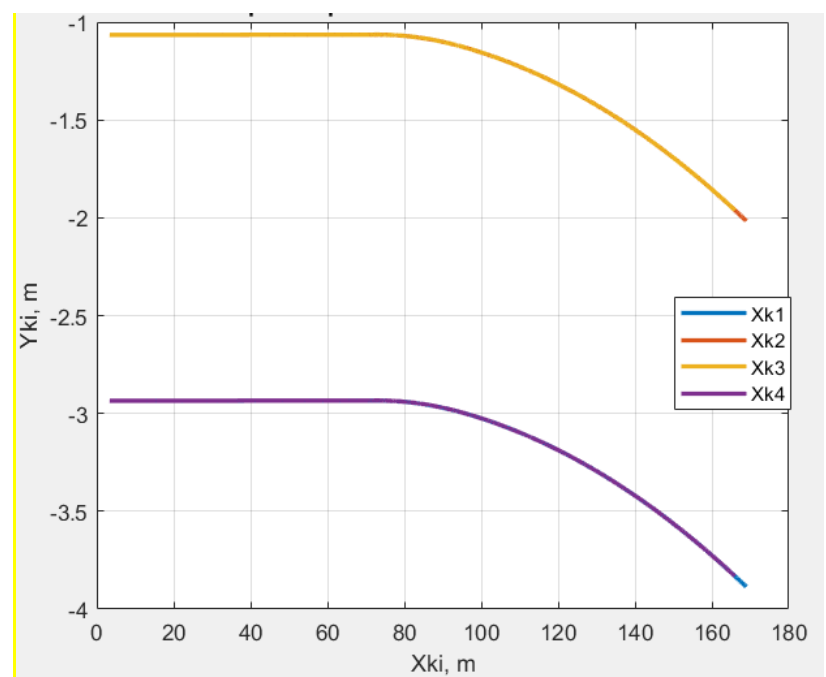

Fig. 23. Trajectory of the centers of the wheels.

4. In the event of a sudden technical tire failure with a complete blowout, there is a change in the direction of motion. The adjustment of the vehicle heading should be smooth to a complete stop. Otherwise, loss of control and instability may occur. 
Hristo Uzunov, Silvia Dechkova, Kaloyan Dimitrov and Vasil Uzunov/

Journal of Engineering Science and Technology Review 14 (4) (2021) 61 - 68

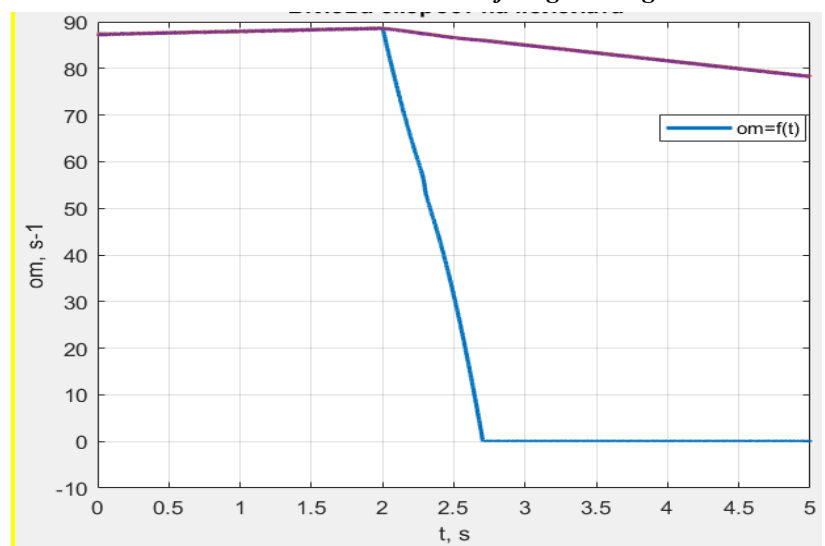

Fig. 24. Angular velocity of wheels $\dot{\gamma}_{i}(i=1 \div 4)$.

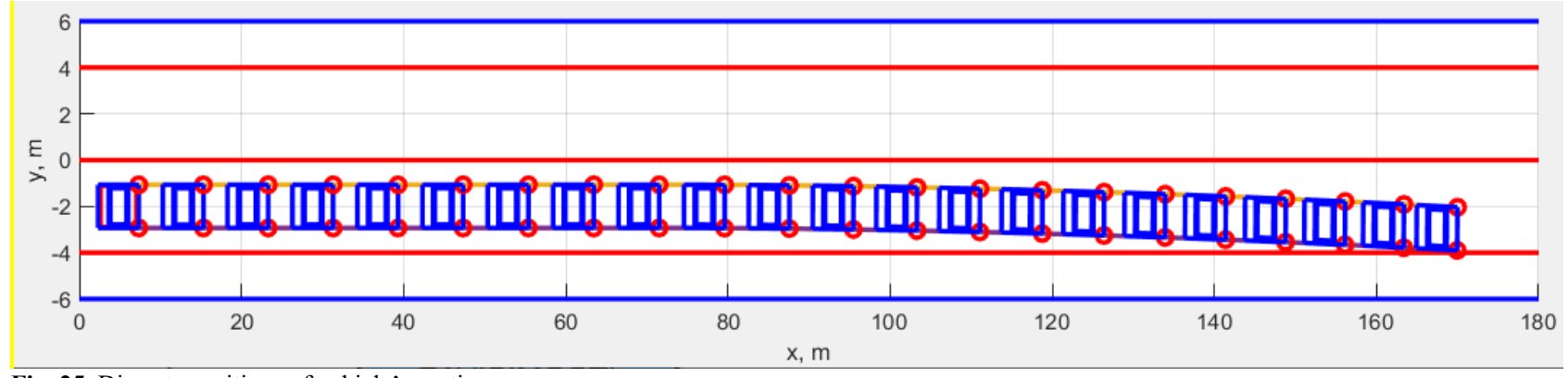

Fig. 25. Discrete positions of vehicle's motion.

\section{Acknowledgements}

The author/s would like to thank the Research and Development Sector at the Technical University of Sofia for the financial support.
This is an Open Access article distributed under the terms of the Creative Commons Attribution License.

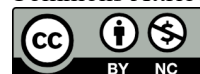

\section{References}

1. Daily, J., N. Shigemura, J. Daily. Fundamentals of Traffic Crash Reconstruction. Jacksonville, Florida: Institute of Police Technololgy and Managment, University of North Florida (2006).

2. Schmidt, B., W. Haight, T. Szabo, J. Welcher. System-based energy and momentum analysis of collisions, in Accident Reconstruction: Technology and Animation VIII SP-1319, no. 980026, Society of Automotive Engineers, Warrendale, PA, February, (1998).

3. Sharma, D., S. Stern, J. Brophy. An Overview of NHTSA's Crash Reconstruction Software WinSMASH. In Proceedings of the Twentieth International Conference on Enhanced Safety of Vehicles, Paper No. 070211, Lyon, France, (2007).

4. Wach, W. Analiza deformacji samochodu według standardu CRASH3. Część 2: Pomiar głębokości odkształcenia (Analysis of motor vehicle deformation according to the CRASH3 standard. Part 2: Measurement of deformation depth). Paragraf na Drodze No. 12, (2003).

5. Jiang, T., Grzebieta, R. H., Rechnitzer, G., Richardson, S., Zhao, X. L. Review of Car Frontal Stiffness Equations for Estimating Vehicle Impact Velocities. The 18th International Technical Conference on the Enhanced Safety of Vehicles Conference (ESV), Nagoya, (2003).

6. Niehoff, P., C. Gabler. The Accuracy of WinSMASH Delta-V Estimates: The Influence of Vehicle Type, Stiffness, and Impact Mode." In the 50th Annual Proceedings of the Association for the Advancement of Automotive Medicine, Annu Proc Assoc Adv Automot Med. 2006; 50: 73-89, Chicago, IL, (2006).
7. Owsiański, R. Szacowanie energii deformacji nadwozi kompaktowych samochodów osobowych (Estimation of the bodywork deformation energy of compact passenger cars). Paragraf na Drodze No.4, (2007).

8. Stronge, W. Impact Mechanics. Cambridge: Cambridge University Press, (2000).

9. Dechkova, S. Creation of multi-mass models in the SolidWorks and Matlab environment for crash identification. Machine Mechanics №119, ISSN 0861-9727, pp. 28 - 32, Bulgaria, (2018).

10. Karapetkov, S. Auto Technical Expertise, Technical University - Sofia, Bulgaria (2005).

11.Karapetkov, S. Investigation of road traffic accident, technical commentary on the lawyer. Technical University of Sofia, Bulgaria, (2010).

12. Karapetkov, S., H. Uzunov. Dynamics of transverse resistance of a car. Didada Consult - Sofia, Bulgaria (2016).

13. Karapetkov, S., L. Dimitrov, H. Uzunov, S. Dechkova. Identifying Vehicle and Collision Impact by Applying the Principle of Conservation of Mechanical Energy. Transport and Telecommunication Riga, volume 20, no. 3, pp. 191-204, doi 10.2478/ttj-2019-0016, Latvia, (2019).

14. Karapetkov, S., L. Dimitrov , H. Uzunov, S. Dechkova. Examination of vehicle impact against stationary roadside objects. 9th International Scientific Conference on Research and Development of Mechanical Elements and Systems, IRMES 2019, University of Kragujevac, Faculty of EngineeringKragujevac; Serbia; 5 September 2019 through 7 September 2019; Code 154497, Kragujevac, Serbia, (2019). 